\title{
1 ABA represses TOR and root meristem activity through nuclear exit of the SnRK1 kinase
}

2 Borja Belda-Palazón ${ }^{1 *}$, Mónica Costa ${ }^{1,2,3}$, Tom Beeckman ${ }^{2,3}$, Filip Rolland ${ }^{4}$, Elena Baena3 González ${ }^{1 *}$

4

$5{ }^{1}$ Instituto Gulbenkian de Ciência, 2780-156 Oeiras, Portugal and GREEN-IT Bioresources for

6 Sustainability, ITQB NOVA, 2780-157 Oeiras, Portugal

$7 \quad{ }^{2}$ Department of Plant Biotechnology and Bioinformatics, Ghent University, Technologiepark 71,

8 B-9052 Ghent, Belgium

$9 \quad{ }^{3}$ VIB-UGent Center for Plant Systems Biology, Technologiepark 71, B-9052 Ghent, Belgium

$10{ }^{4}$ Laboratory for Molecular Plant Biology, Biology Department, Katholieke Universiteit Leuven, 113001 Heverlee-Leuven, Belgium

12

13

$14{ }^{*}$ Corresponding authors. Instituto Gulbenkian de Ciência, Rua da Quinta Grande 6, 2780-156 15 Oeiras, Portugal. Tel.: +351 214464630; Fax: +351 214407970; e-mail:

16 bbelda@igc.gulbenkian.pt, ebaena@igc.gulbenkian.pt

19 Keywords: root apical meristem, growth regulation, abscisic acid, energy signaling, SnRK1, TOR, 20 Arabidopsis thaliana 


\section{ABSTRACT}

23 The phytohormone abscisic acid (ABA) promotes plant tolerance to major stresses like drought, 24 partly by modulating plant growth and development. However, the underlying mechanisms are 25 poorly understood. Here, we show that cell proliferation in the Arabidopsis thaliana root meristem 26 is controlled by the interplay between three kinases, SNF1-RELATED KINASE 2 (SnRK2), the 27 main driver of ABA signaling, the SnRK1 energy sensor, and the growth-promoting TARGET OF

28 RAPAMYCIN (TOR) kinase. Under favorable conditions, the SnRK1 1 1 catalytic subunit is 29 enriched in the nuclei of root meristematic cells and this is accompanied by normal cell 30 proliferation and meristem size. Depletion of SnRK2s in a snrk2.2 snrk2.3 double mutant causes 31 constitutive cytoplasmic localization of $\operatorname{SnRK} 1 \alpha 1$ and a reduction in meristem size, suggesting 32 that, under non-stress conditions, SnRK2s enable growth by retaining SnRK1 $\alpha 1$ in the nucleus. In

33 response to elevated ABA levels, SnRK1 $\alpha 1$ translocates to the cytoplasm and this is accompanied 34 by inhibition of TOR, decreased cell proliferation and meristem size. Blocking nuclear export with 35 leptomycin B abrogates ABA-driven SnRK1 $\alpha 1$ relocalization to the cytoplasm and the inhibition 36 of TOR. Fusion of SnRK1 $\alpha 1$ to an SV40 nuclear localization signal leads to defective TOR 37 repression in response to $\mathrm{ABA}$, demonstrating that SnRK1 $1 \alpha 1$ nuclear exit is a premise for this 38 repression. Finally, the SnRK2-dependent changes in SnRK1 $\alpha 1$ subcellular localization are 39 specific to the proliferation zone of the meristem, underscoring the relevance of this mechanism 40 for growth regulation. 
42 The phytohormone abscisic acid (ABA) plays major roles in plant stress responses. ABA signals

43 are transduced through a well-established pathway whose main effectors in Arabidopsis are SNF1-

44 RELATED PROTEIN KINASE 2.2 (SnRK2.2), SnRK2.3, and SnRK2.6 (1). ABA promotes plant

45 adaptation partly by modifying developmental programs, having a major impact e.g. on root

46 architecture (2). ABA modulates primary root (PR) and lateral root (LR) growth through

47 interactions with other hormones, ultimately affecting cell division and elongation by poorly

48 understood mechanisms (2). We recently uncovered an intimate connection between ABA and

49 SnRK1 signalling that is crucial for shaping root architecture in a TARGET OF RAPAMYCIN

50 (TOR)-dependent manner (3). TOR is a protein kinase complex that promotes cell proliferation

51 (4). It is highly enriched in the meristems and its inactivation causes reduced root meristem size

52 and defective PR growth (4). SnRK1 is an evolutionarily conserved protein kinase complex that is

53 activated when energy levels decline during stress, conferring protection partly by limiting growth

54 (5). SnRK1 is also activated by ABA, enabling plants to repress growth when water is scarce (3).

55 Under favorable conditions, the main catalytic subunit SnRK1 $\alpha 1$ is sequestered by SnRK2-

56 containing repressor complexes, allowing TOR to be active. In response to ABA, these complexes

57 dissociate, releasing SnRK2 and SnRK1 $\alpha 1$, which inhibits TOR and growth (3). Consistent with

58 this model, the snrk2.2 snrk2.3 mutant (snrk2d) shows markedly defective PR growth under

59 favorable conditions due to aberrant repression of TOR activity (3). This defect is fully rescued by

60 the snrk1 $\alpha 1$ mutation, demonstrating it is SnRK1 1 1-dependent (3).

To investigate further how TOR and root growth are controlled by SnRK2 and SnRK1, we examined the root meristems of Col-0 control seedlings, the snrk2d mutant, and its cross with

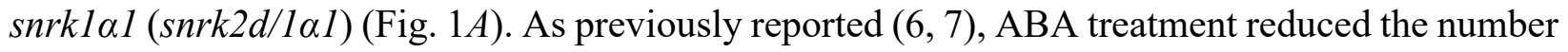

64 of root meristematic cells, leading to smaller meristems in Col-0. In contrast, snrk2d showed a reduction in meristem size and cell number already under mock conditions, and this was fully

66 rescued by the snrklal mutation. Consistent with the ABA hyposensitivity of snrk2d and snrk2d/1 $\alpha 1$ (3), the addition of ABA did not further decrease meristem size or cell number in these mutants (Fig. 1A). These meristem phenotypes correlate well with the PR length previously observed in these mutants and conditions (3) and suggest that reduced cell proliferation contributes 
We next hypothesized that regulation of SnRK1 and growth by ABA and SnRK2s could involve changes in SnRK1al subcellular localization. Firstly, SnRK1 and SnRK2 are highly enriched in the nuclei of root meristematic cells (3). Secondly, SnRK2-harboring SnRK1 repressor complexes localize to the nucleus in Nicotiana benthamiana epidermal cells (3). Thirdly, in planta, the TOR complex subunit RAPTOR1B interacts with $\operatorname{SnRK} 1 \alpha 1(3,8)$ in the cytosol (9). Fourthly, the subcellular localization of SnRK1 $1 \alpha 1$ is central to its function (10). We therefore monitored SnRK $1 \alpha 1$ subcellular localization in mock- or ABA-treated seedlings in two independent lines

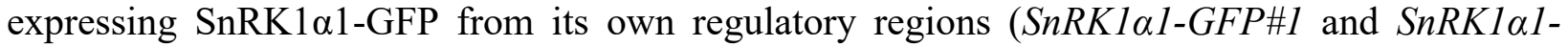
$G F P \# 2)$. SnRK $1 \alpha 1$ was detected in the meristematic, elongating and differentiating zones of the PR both in mock and ABA, displaying a known nuclear and cytoplasmic localization (11) (Fig. $1 B$ ). In mock, SnRK1 $\alpha 1$ exhibited a ring-shaped pattern characteristic of nuclear proteins that are absent from the nucleolus in meristematic cells (12) (Fig. 1B). In ABA, the nuclear signal appeared to decline specifically in the meristematic zone (Fig. 1B). Electron micrographs confirmed that a ring-shaped nuclear region surrounds a large-sized nucleolus in these cells (Fig. 1C). Closer examination and GFP quantification in the meristematic zone revealed overall similar SnRK1 $\alpha 1$ GFP levels in mock- and ABA-treated roots but a reduction in the nucleus to cytoplasm ratio (N/C) from 1.19 (line \#1) and 1.2 (line \#2) in mock to 0.73 (line \#1) and 0.71 (line \#2) in ABA (Fig. 1D), suggesting that ABA induces SnRK1al relocalization from the nucleus to the cytoplasm. Accordingly, the ABA effect was fully abolished by the nuclear export inhibitor Leptomycin B $(\mathrm{LMB} ; \mathrm{N} / \mathrm{C}$ ratio=1.36 and 1.23 in lines \#1 and \#2; Fig. 1D).

Given the marked reduction in meristem size (Fig. 1A) and PR length (3) of the snrk2d mutant, we investigated the role of SnRK2s in SnRK1 1 1 localization using the cross of snrk2d with the SnRK1 $\alpha 1-G F P \# 2$ line (Fig. 1E). In mock, SnRK1 $\alpha 1-$ GFP was barely present in the nuclei of snrk $2 d$ meristematic cells, with most of the GFP signal being cytoplasmatic and yielding a N/C ratio of 0.75 (Fig. 1E), comparable to the ABA-treated SnRK1 $\alpha 1-G F P \# 2$ control (Fig. 1D). SnRK2.2-GFP suffered from overall protein degradation, but there was no change in its subcellular

97 localization (Fig. 1F). Nuclear exit of SnRK1 $\alpha 1$ could be observed in other highly proliferating 98 regions of the root, such as the LR tip (Fig. 1G). However, in differentiated root cells, ABA treatment and SnRK2 depletion did not alter SnRK1 $\alpha 1$ subcellular localization (Fig. 1H).

100 Furthermore, the ABA-triggered changes in SnRK1 $\alpha 1$ localization remained undetected in 101 nucleus/cytoplasm fractions of whole roots (Fig. 1I). Altogether, these results indicate that the 
102 SnRK2-dependent localization of SnRK1 $\alpha 1$ and its ABA-triggered nuclear exit is specific to

103 meristematic cells, consistent with reports that SnRK1 $\alpha 1$ subcellular localization is controlled in 104 a cell type-specific manner $(13,14)$.

105

106

107

108

109

110

111

112

113

Given that SnRK1 $1 \alpha 1$ is required for the inhibitory effect of ABA on TOR and growth (3), we wondered whether SnRK1 $1 \alpha 1$ nuclear exit is a premise for such inhibition. Indeed, pre-treatment with LMB blocked the ABA-triggered repression of TOR activity (Fig. 2A), measured as phosphorylation of ribosomal protein S6 [RPS6 $\left.{ }^{\mathrm{S} 240} ;(3)\right]$. Moreover, as compared to control plants expressing wild-type SnRK1 $\alpha 1$ (control- $\alpha 1$ ), the repression of TOR by ABA was also defective when SnRK1 $\alpha 1$ was fused to an SV40 nuclear localization sequence (NLS) that favors its presence in the nucleus (NLS- $\alpha 1$; Fig. $2 B$ ). This demonstrates that SnRK $1 \alpha 1$ nuclear exit is necessary for inhibiting TOR in response to ABA. Furthermore, the fact that nuclear export is crucial for repressing TOR (Fig. 2A) but only SnRK1 $\alpha 1$ (Fig. 1D), and not SnRK2.2, translocates to the cytoplasm in response to the hormone (Fig. $1 F$ ), suggests that the previously reported role of SnRK2s in this process $(3,15)$ may be indirect via SnRK1 1 1 regulation.

We conclude that root growth is modulated by ABA through changes in SnRK1 $\alpha 1$ subcellular localization, allowing control of TOR activity and cell proliferation in the root meristem in accordance to e.g. water availability (Fig. 2C). When conditions are favorable, SnRK1 $\alpha 1$ is sequestered in the nucleus by SnRK2-containing repressor complexes. Dissociation of these complexes in response to ABA releases SnRK1 1 1, which translocates to the cytoplasm and inhibits TOR activity and growth. This mode of regulation appears to be specific to meristematic cells, where TOR is highly enriched and where active cell proliferation takes place (4). Future work will address whether such specificity stems from the exclusive ability of meristematic cells to form SnRK2-SnRK1 $\alpha 1$ complexes, to export SnRK1 11 from the nucleus or both.

\section{REFERENCES}

1. S. R. Cutler, P. L. Rodriguez, R. R. Finkelstein, S. R. Abrams, Abscisic Acid: Emergence of a Core Signaling Network. Annual Review of Plant Biology 61, 651-679 (2010).

2. J. M. Harris, Abscisic Acid: Hidden Architect of Root System Structure. Plants 4, 548572 (2015).

3. B. Belda-Palazón, et al., A dual function of SnRK2 kinases in the regulation of SnRK1 and plant growth. Nature Plants 6, 1345-1353 (2020). 
133 4. Z. Ahmad, Z. Magyar, L. Bögre, C. Papdi, Cell cycle control by the target of rapamycin signalling pathway in plants. Journal of Experimental Botany 70, 2275-2284 (2019).

5. M. Jamsheer K, M. Kumar, V. Srivastava, SNF1-related protein kinase 1: the many-faced signaling hub regulating developmental plasticity in plants. Journal of Experimental Botany 72, 6042-6065 (2021).

6. S. Promchuea, Y. Zhu, Z. Chen, J. Zhang, Z. Gong, ARF2 coordinates with PLETHORAs and PINs to orchestrate ABA-mediated root meristem activity in Arabidopsis. Journal of Integrative Plant Biology 59, 30-43 (2017).

7. L. Yang, et al., ABA-Mediated ROS in Mitochondria Regulate Root Meristem Activity by Controlling PLETHORA Expression in Arabidopsis. PLOS Genetics 10, e1004791 (2014).

8. J. van Leene, et al., Capturing the phosphorylation and protein interaction landscape of the plant TOR kinase. Nature Plants 5, 316-327 (2019).

9. E. Nukarinen, et al., Quantitative phosphoproteomics reveals the role of the AMPK plant ortholog SnRK1 as a metabolic master regulator under energy deprivation. Scientific Reports 6, 31697 (2016).

10. M. Ramon, et al., Default activation and nuclear translocation of the plant cellular energy sensor SnRK1 regulate metabolic stress responses and development. Plant Cell 31, 1614 1632 (2019).

11. M. Bitrián, F. Roodbarkelari, M. Horváth, C. Koncz, BAC-recombineering for studying plant gene regulation: Developmental control and cellular localization of SnRK1 kinase subunits. Plant Journal 65, 829-842 (2011).

12. B. Desvoyes, A. Arana-Echarri, M. D. Barea, C. Gutierrez, A comprehensive fluorescent sensor for spatiotemporal cell cycle analysis in Arabidopsis. Nature Plants 6, 1330-1334 (2020).

13. C. Han, et al., KIN10 promotes stomatal development through stabilization of the SPEECHLESS transcription factor. Nature Communications 11, 1-10 (2020).

14. N. E. Blanco, D. Liebsch, M. G. Díaz, Å. Strand, J. Whelan, Dual and dynamic intracellular localization of Arabidopsis thaliana SnRK1.1. Journal of Experimental Botany 70, 2325-2338 (2019).

15. P. Wang, et al., Reciprocal Regulation of the TOR Kinase and ABA Receptor Balances Plant Growth and Stress Response. Molecular Cell 69, 100-112.e6 (2018). old seedlings with or without ABA treatment (50 $\mathrm{M}, 48 \mathrm{~h})$. Arrowheads: region for cortical cell number and meristem length quantifications (violin plots). $n=23-24 ; p<0.05$, one-way ANOVA

171 with Tukey's HSD test. Scale bar: $50 \mu \mathrm{m}$. (B) Root apices of 4d-old SnRK1 $\alpha 1-G F P \# 1$ seedlings 172 with or without ABA treatment $(50 \mu \mathrm{M}, 3 \mathrm{~h})$. Scale bar: $30 \mu \mathrm{m}$. Arrowheads: same position 173 between upper and bottom photographs. (C) Electron micrograph of meristematic epidermal cells 
174 with magnification (right panel) showing the cellular ultrastructure. C: cytoplasm; N: nucleus; No: 175 nucleolus. Scale bars: left, $5 \mu \mathrm{m}$; right, $1 \mu \mathrm{m}$. (D-E) SnRK1 $\alpha 1-G F P$ subcellular localization in 176 epidermal cells of 4d-old root meristems. Scale bar: $10 \mu \mathrm{m}$. Dotted lines: nuclear boundary. 177 Quantification of SnRK1 $\alpha 1$-GFP mean cellular fluorescence and nucleus-to-cytosol (N/C) ratios. $178 n=5-6(D)$ or $8(E)$; one-way ANOVA with Dunett's test. $(D)$ Two independent SnRK1 $\alpha 1-G F P$ 179 lines (\#1 and \#2) with or without ABA $(50 \mu \mathrm{M}, 3 \mathrm{~h})$ or LMB $(2.5 \mu \mathrm{M}$, $1 \mathrm{~h}$ prior to ABA addition)

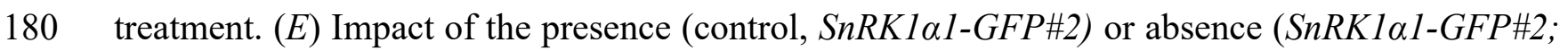
$181 s n r k 2 d)$ of SnRK2s. (F) Expression and subcellular localization of SnRK2.2-GFP in epidermal 182 cells of $4 \mathrm{~d}$-old root meristems treated and quantified as in $(D)$. Scale bar: $10 \mu \mathrm{m}$. $n=5$; two-tailed 183 Student t-test. $(G-H)$ SnRK1 $\alpha 1$-GFP subcellular localization in LRs $(G)$ and the PR differentiation 184 zone $(H)$ in seedlings (Line \#1 and/or \#2) with or without ABA treatment $(50 \mu \mathrm{M}, 3 \mathrm{~h})$ and in 185 absence (SnRK1a1-GFP\#2; snrk2d) of SnRK2s. Scale bar: $30 \mu \mathrm{m}$. $(G)$ Nine-day old seedlings. $186(H)$ Four-day old seedlings. N/C ratios of differentiated root cells. $n=5$, two-tailed Student t-test 187 (line \#1) or one-way ANOVA (line \#2). PI, propidium iodide. $n s$, non-significant. (I) 188 Representative SnRK1 $\alpha 1$ immunoblot from nuclear and cytoplasmic fractions of 14d-old Col-0 189 whole roots with or without ABA (50 $\mu \mathrm{M}$ ABA, 3h). H3, Histone 3 marker for nuclear fractions.

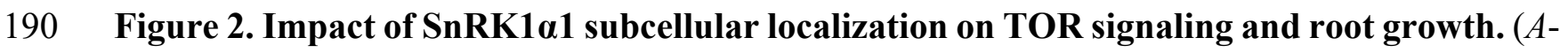

191 B) Representative immunoblots and quantification of RPS6 ${ }^{\text {S240 }}$ phosphorylation (phospho192 RPS6/total-RPS6) in Col-0 (A) or control- $\alpha 1$ and NLS- $\alpha 1$ (B) seedlings with or without ABA (50 $193 \mu \mathrm{M}, 3 \mathrm{~h})$ or LMB $(2.5 \mu \mathrm{M}, 1 \mathrm{~h}$ prior to ABA addition). Images from the same gel were cropped for 194 showing $\alpha 1$ and NLS- $\alpha 1$ contiguously. $(A) n=5 ;(B) n=3$; error bars, SEM; two-tailed Student $t$ 195 test. (C) Under favorable conditions, SnRK1 $1 \alpha 1$ is sequestered in the nucleus by repressor 196 complexes containing SnRK2 [and a PP2C (3)], enabling TOR activity in the cytoplasm, 197 meristematic cell proliferation and root growth. Dissociation of these complexes in ABA by the 198 hormone-bound PYR/PYL/RCAR receptors releases SnRK1 $\alpha 1$ which exits the nucleus and 199 inhibits TOR and growth. TORC1, TOR Complex 1; $N$, nucleus; $C$, cytoplasm. Created with 200 BioRender.com.

\section{Materials and Methods}

202 Experimental details are provided in SI Appendix. 
203 All study data are included in the paper.

\section{Acknowledgements}

205 We thank J-K. Zhu, M. Bennett and C. Koncz for snrk2d, SnRK2.2-GFP, and SnRK1 1 1-GFP\#1

206 seeds. We also thank the IGC Facilities for excellent plant care (Plant), sample processing and

207 imaging (Electron Microscopy) and technical advice (Advanced Imaging; supported by PPBI-

208 POCI-01-0145-FEDER-022122). This work was funded by FCT [UIDB/04551/2020, LISBOA-

209 01-0145-FEDER-028128, PTDC/BIA-BID/32347/2017], and by EU Horizon 2020 programme

210 (BBP; H2020-WF-2018-2020/H2020-WF-01-2018, Grant 867426).

\section{Author contributions}

212 BBP performed experiments and analyzed data. MC performed the root meristem assays and 213 analyzed the data. TB and FR contributed tools and expertise and supported conceptual work. EBG 214 and BBP conceived the project, designed experiments, analyzed and interpreted data and wrote the 215 manuscript.

\section{Competing interests}

217 The authors declare no competing interests. 

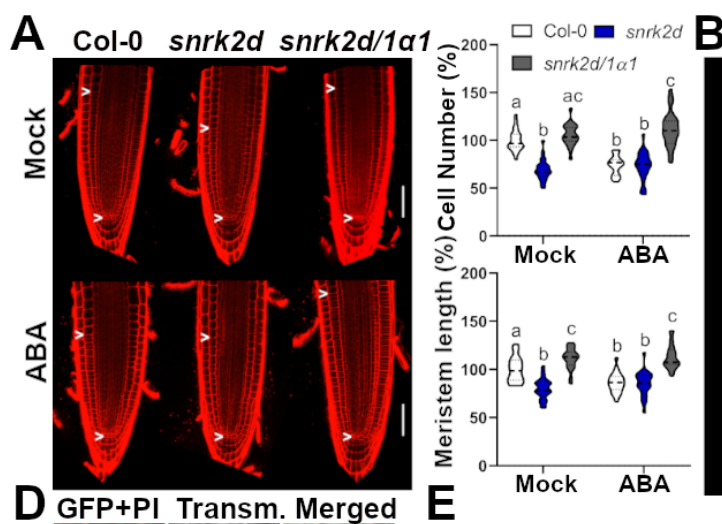

B SnRK1a1-GFP\#1 C
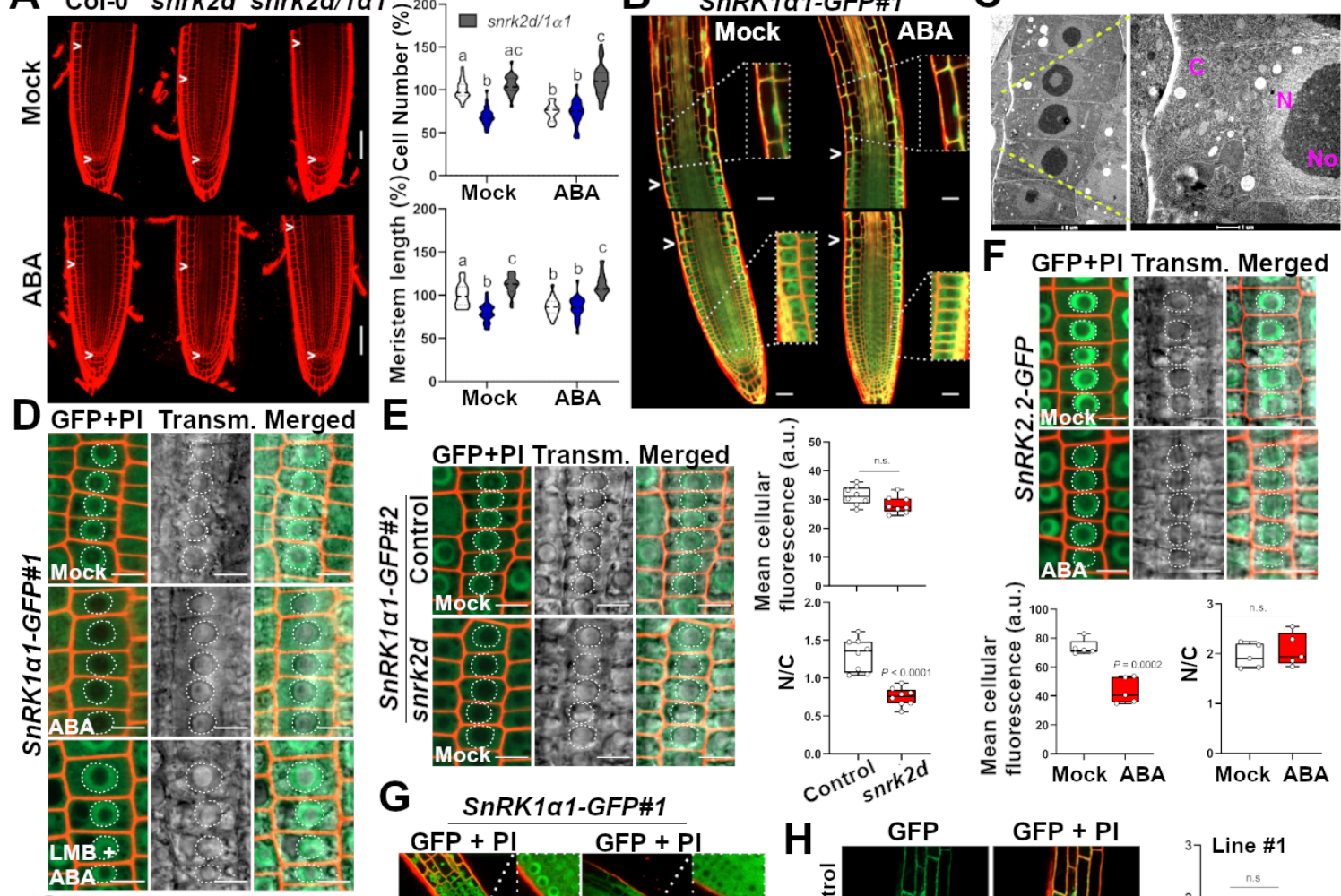

\section{G}
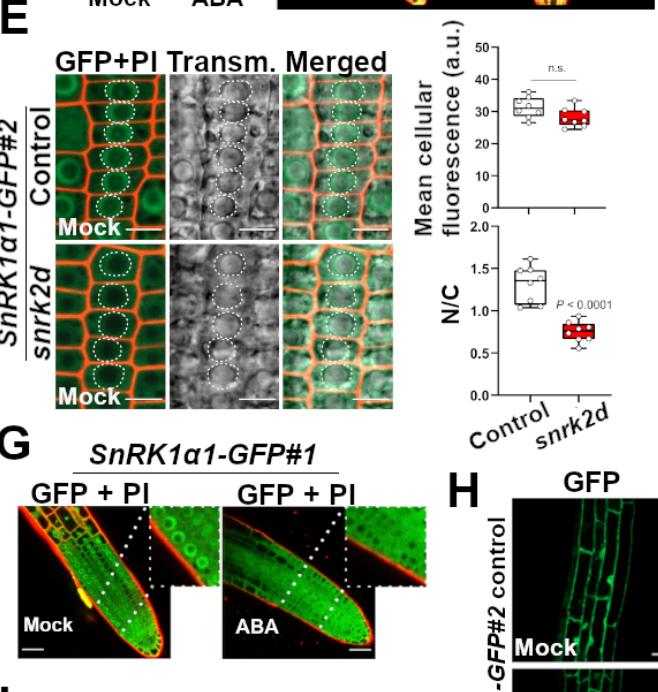

F GFP+PI Transm. Merged

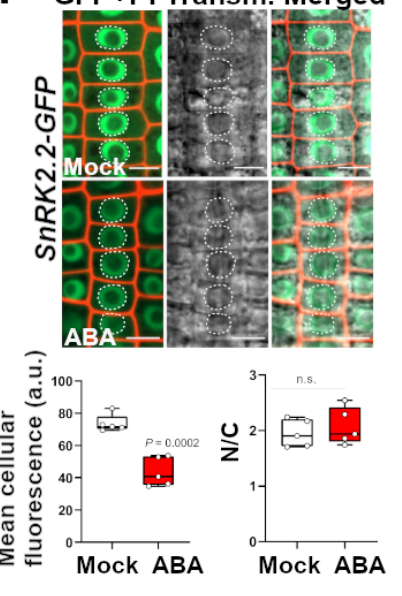

I

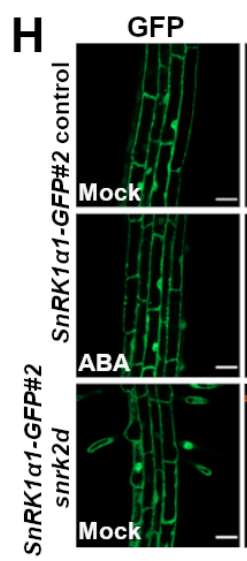

GFP + PI
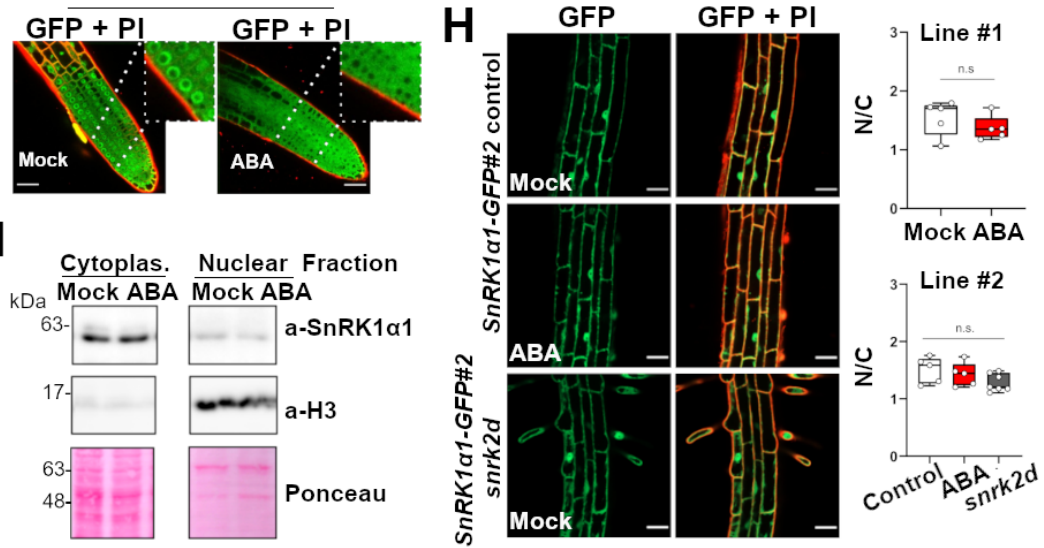

Figure 1. Impact of ABA and SnRK2s on SnRK1a1 subcellular localization and cell proliferation in the root apical meristem. (A) Meristems of Col-0, snrk2d and snrk2d/1 $\alpha 1$ 7d-old seedlings with or without ABA treatment $(50 \mu \mathrm{M}, 48 \mathrm{~h})$. Arrowheads: region for cortical cell number and meristem length quantifications (violin plots). $n=23-24 ; p<0.05$, one-way ANOVA with Tukey's HSD test. Scale bar: $50 \mu \mathrm{m}$. (B) Root apices of 4d-old SnRK1 $\alpha 1$-GFP\#1 seedlings with or without ABA treatment $(50 \mu \mathrm{M}, 3 \mathrm{~h})$. Scale bar: $30 \mu \mathrm{m}$. Arrowheads: same position between upper and bottom photographs. $(C)$ Electron micrograph of meristematic epidermal cells with magnification (right panel) showing the cellular ultrastructure. C: cytoplasm; N: nucleus; No: nucleolus. Scale bars: left, $5 \mu \mathrm{m}$; right, $1 \mu \mathrm{m}$. (D-E) SnRK1 $\alpha 1-$ GFP subcellular localization in epidermal cells of 4dold root meristems. Scale bar: $10 \mu \mathrm{m}$. Dotted lines: nuclear boundary. Quantification of SnRK1 $\alpha 1-G F P$ mean cellular fluorescence and nucleus-to-cytosol (N/C) ratios. $n=5-6(D)$ or $8(E)$; one-way ANOVA with Dunett's test. $(D)$ Two independent SnRK1 $\alpha 1-$ GFP lines (\#1 and \#2) with or without ABA $(50 \mu \mathrm{M}, 3 \mathrm{~h})$ or LMB $(2.5 \mu \mathrm{M}$, 1h prior to ABA addition) treatment. $(E)$ Impact of the

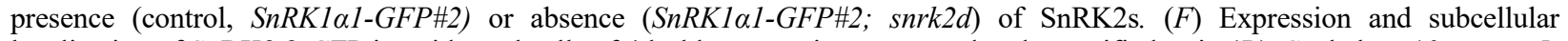
localization of SnRK2.2-GFP in epidermal cells of 4d-old root meristems treated and quantified as in $(D)$. Scale bar: $10 \mu \mathrm{m} . n=5$; two-tailed Student t-test. $(G-H)$ SnRK1 $\alpha 1$-GFP subcellular localization in LRs $(G)$ and the PR differentiation zone $(H)$ in seedlings (Line \#1 and/or \#2) with or without ABA treatment $(50 \mu \mathrm{M}, 3 \mathrm{~h})$ and in absence (SnRK1 1 1-GFP\#2; snrk2d) of SnRK2s. Scale bar: $30 \mu \mathrm{m}$. $(G)$ Nine-day old seedlings. $(H)$ Four-day old seedlings. N/C ratios of differentiated root cells. $n=5$, two-tailed Student ttest (line \#1) or one-way ANOVA (line \#2). PI, propidium iodide. $n s$, non-significant. (I) Representative SnRK1 $\alpha 1$ immunoblot from nuclear and cytoplasmic fractions of $14 \mathrm{~d}$-old Col- 0 whole roots with or without ABA (50 $\mu \mathrm{M} \mathrm{ABA}, 3 \mathrm{~h})$. H3, Histone 3 marker for nuclear fractions. 

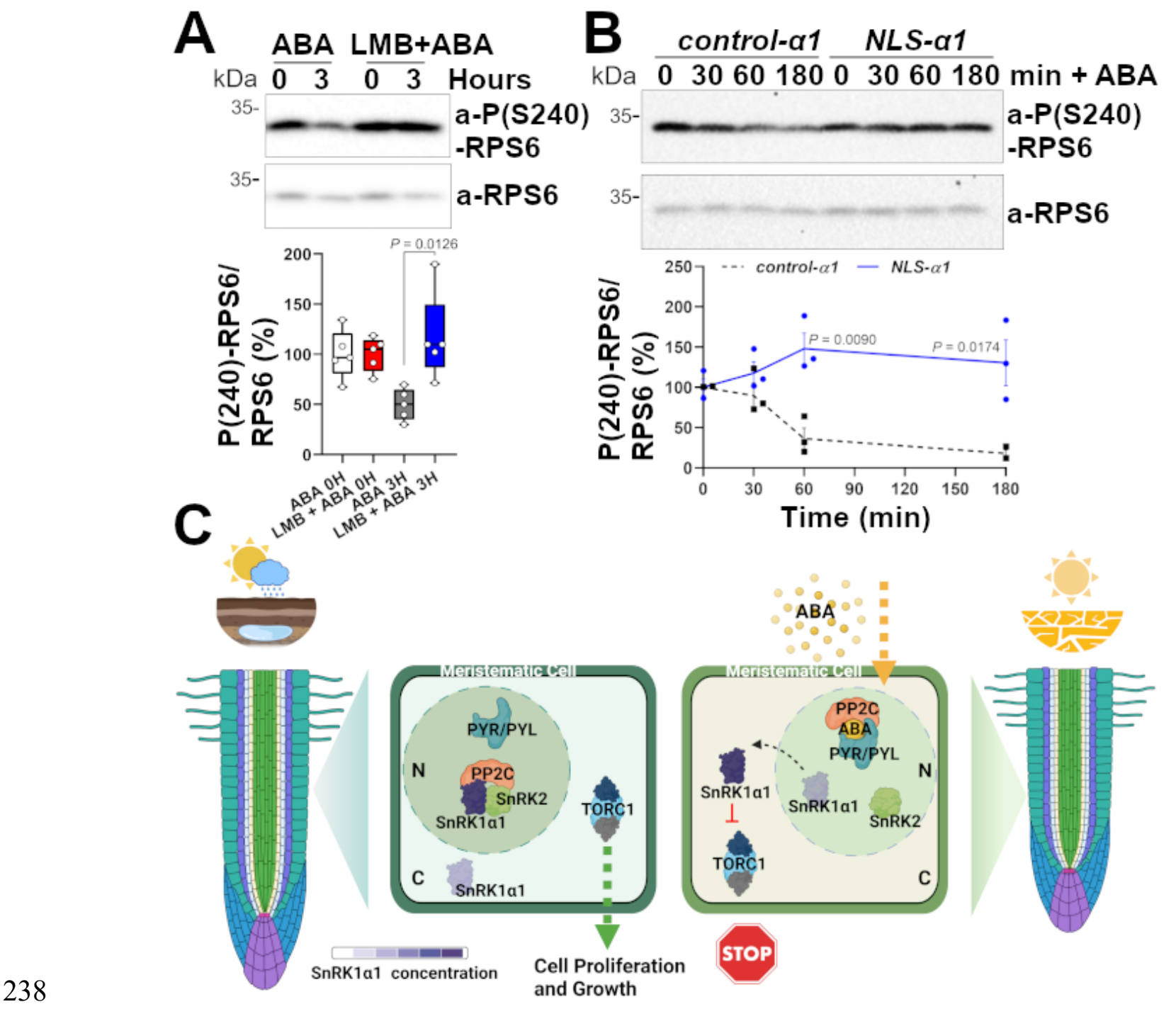

Figure 2. Impact of SnRK1a1 subcellular localization on TOR signaling and root growth. $(A-B)$ Representative immunoblots and quantification of RPS6 ${ }^{\mathrm{S} 240}$ phosphorylation (phospho-RPS6/total-RPS6) in Col-0 (A) or control- $\alpha 1$ and $N L S-\alpha 1$ (B) seedlings with or without $\mathrm{ABA}(50 \mu \mathrm{M}, 3 \mathrm{~h})$ or $\mathrm{LMB}(2.5 \mu \mathrm{M}, 1 \mathrm{~h}$ prior to $\mathrm{ABA}$ addition). Images from the same gel were cropped for showing $\alpha 1$ and $N L S$ - $\alpha 1$ contiguously. $(A) n=5 ;(B) n=3$; error bars, SEM; two-tailed Student $t$-test. (C) Under favorable conditions, SnRK1 $\alpha 1$ is sequestered in the nucleus by repressor complexes containing SnRK2 [and a PP2C (3)], enabling TOR activity in the cytoplasm, meristematic cell proliferation and root growth. Dissociation of these complexes in ABA by the hormone-bound PYR/PYL/RCAR receptors releases SnRK1 $\alpha 1$ which exits the nucleus and inhibits TOR and growth. TORC1, TOR Complex 1; $N$, nucleus; $C$, cytoplasm. Created with BioRender.com. 